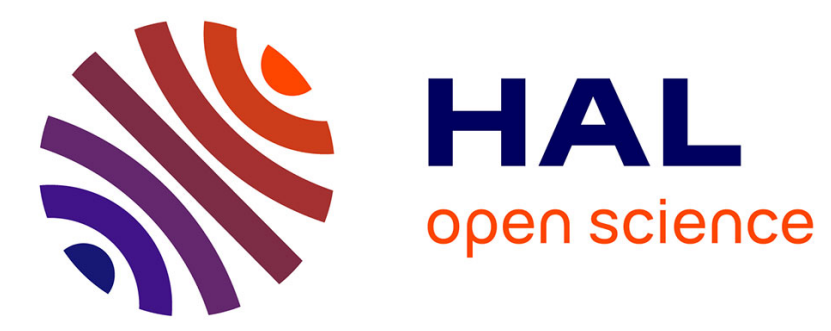

\title{
L'évolutionnisme bien-pensant ou l'ethnologie à sens unique
}

Hélène Claudot-Hawad

\section{To cite this version:}

Hélène Claudot-Hawad. L'évolutionnisme bien-pensant ou l'ethnologie à sens unique. Cahiers d'études africaines, 1994, XXXIV (136), pp.673-685. halshs-00604632v2

\section{HAL Id: halshs-00604632 \\ https://shs.hal.science/halshs-00604632v2}

Submitted on 1 Jul 2011

HAL is a multi-disciplinary open access archive for the deposit and dissemination of scientific research documents, whether they are published or not. The documents may come from teaching and research institutions in France or abroad, or from public or private research centers.
L'archive ouverte pluridisciplinaire HAL, est destinée au dépôt et à la diffusion de documents scientifiques de niveau recherche, publiés ou non, émanant des établissements d'enseignement et de recherche français ou étrangers, des laboratoires publics ou privés. 


\title{
L'évolutionnisme bien-pensant ou l'ethnologie à sens unique
}

\author{
Hélène Claudot-Hawad
}

Résumé :

L'interprétation du soulèvement armé touareg des années 1990 a donné lieu à de violentes polémiques qui révèlent les rapports étroits noués entre science et pouvoir en temps de crise et les tentatives d'interdire tout discours contrariant les thèses étatiques officielles. Réponse ici aux attaques ciblées de Bourgeot et Casajus contre mes analyses, politiquement incorrectes, de la situation touarègue qui a conduit à l'insurrection de 1990 au Niger et au Mali.

\footnotetext{
"Répondons très simplement, comme répondrait, j'imagine, notre innocent frère mythique dans son village vierge poursuivant ses jeux innocents et soudain face à face avec le personnage de Descartes en casque colonial occupé à pénétrer la jungle de la mentalité noire prélogique avec sa pirogue intellectuelle. Lorsque notre fantôme cartésien se présente en griffonnant sur la tabula rasa (naturellement) de notre frère noir la célèbre proposition : "je pense, donc je suis", nous ne devons pas répondre, comme le firent les Négritudinistes : “je sens, donc je suis”, car ce serait accepter l'arrogance d'une certitude philosophique sans fondement démontrable et qui réduit la logique cosmique de l'être à un particularisme fonctionnel de l'être. Je ne puis imaginer que notre "authentique innocent noir" se laisserait jamais manipuler au point d'adopter la position fausse qui consiste à opposer un pernicieux manichéisme à un autre. Je soupçonne qu'il préfèrerait réduire notre explorateur blanc à de justes proportions syntaxiques en répondant : "Tu penses, donc tu es un penseur. Tu es quelqu'un-qui-pense, créature-blanche-en-casque-colonial-dans-lajungle-africaine-qui-pense et, finalement, homme-blanc-qui-a-des-difficultés-à-croire-à-ta-propreexistence."

(W. Soyinka, Ideology and the social vision (2) : The secular ideal, in Myth, Literature and the African World, Cambridge University Press, 1979, p. 138, traduit et cité par E. Galle 1987)
} 
Selon un schéma identique, bien qu'en des termes nettement plus policés, A. Bourgeot réitère les critiques déjà formulées dans la revue Politique africaine à l'encontre de mes analyses sur le monde touareg à propos des événements de 1990-1992 (Bourgeot 1992). Lui répondre une nouvelle fois (Claudot-Hawad 1993a), alors que ses arguments sont inchangés et que le débat est absent, a-t-il un sens?

Ma grand-mère appellerait cela "répépiller" mais les grand-mères sont des individualités non signifiantes dans cette polémique qui se veut intellectuelle et on ne sait pas, dirait Bourgeot, à quelle réalité historique et sociologique une telle notion philosophico-mystico-provençalo-française renvoie.

Reprenons donc les faits concrets. Dans la presse, dans les revues scientifiques, dans les enceintes de l'université ou dans celles du CNRS, A. Bourgeot et son allié intellectuel du moment, D. Casajus, se dévouent corps et âme pour dénoncer, à l'aide d'accusations similaires, les dangers de ma démarche, son caractère idéologique, extrémiste, partisan, qui entre autres “noircit à l'extrême la période coloniale et celle de l'indépendance, au profit d'une période précoloniale idyllique, omettant d'intégrer dans (m)es analyses les querelles et guerres incessantes que tous les observateurs ont notées" (Bourgeot ci-dessus). Pour avoir consacré plusieurs articles aux relations de violence chez les Touaregs et, d'autre part, les avoir commentés dans différentes situations historiques (voir en particulier Claudot-Hawad et Hawad 1982 ; Claudot-Hawad 1990, 1993c), ces remarques ont de quoi m'étonner.

Il est vrai que la lecture de mes travaux par Bourgeot repose sur une telle cécité intellectuelle - volontaire ou non - et une mauvaise foi si caricaturale que le sens de mes analyses est systématiquement tronqué. Pour invalider mon travail, Bourgeot agrémente ce procédé d'un postulat essentiel à sa démonstration qui consiste à affirmer que “j'invente". C'est la méthode Faurisson. Il reste à prouver l'essentiel, c'est-à-dire la non existence de mes interlocuteurs touaregs, l'absence des enregistrements que j'ai effectués auprès d'eux, l'irréalité des matériaux et des observations qui ont fait l'objet de multiples publications et sur lesquels je fonde mes analyses, la vacuité de mes nombreuses enquêtes menées de 1982 à 1990 chaque hiver pendant deux à quatre mois, non pas chez de petits groupes "indéterminés" mais toujours nommés et situés, tels que les Idnan, les Taghat Mellet et les Ifoghas dans l'Adagh, les Imedédaghen, les Igawdaren, les Kel Intessar dans l'Awza et le Gourma, les Iwellemeden de Menaka dans 
l'Azawagh occidental, les Touaregs de l'Udalen et enfin les Ikazkazen de l'Aïr. Faute de démonstration crédible, j'en concluerai que Bourgeot défend à travers ses attaques calomnieuses des intérêts qui n'ont rien à voir avec la science.

\section{Vérité et contre-vérités}

Au chapelet de "contre-vérités" dont il me gratifie et que je vais discuter plus loin, Bourgeot oppose son interprétation qui serait la seule valable sur le plan scientifique, comme si la vérité en histoire se réduisait à un schéma interprétatif unique, monolithique immobile, défini une fois pour toutes. Cette conception totalitaire rejette nécessairement les points de vue dissidents aux marges de la science, dans "l'invention", la "falsification" (Bourgeot et Casajus, 1992 ; Salifou, 1993 : 92 ; Hamani, 1994), 1"'affabulation", le “mensonge" (Présidence de la République du Niger, 1994), le "fantasme" (Hamani 1994), "l'idéologie" (Bourgeot 1992), la "forgerie" (Bourgeot ci-dessus), interdisant que se manifestent les interprétations plurielles à partir desquelles s'élaborent la démarche et les hypothèses critiques de l'histoire.

Finalement, où souhaite en venir si ardemment Bourgeot? A prouver que les Touaregs n'ont jamais eu ni pensée ni organisation politiques jusqu'à ce qu'ils aient affaire aux Etats-nations. Ces primitifs isolés et dispersés, qui ne savaient se concevoir qu'en termes tribaux et parentaux, se seraient alors réveillés de leur long sommeil conceptuel et auraient enfin découvert les notions de peuple, de nation, de fédération, de révolution, d'organisation politique, de démocratie... Leur résistance à l'occupation coloniale serait due à l'esprit "libertaire" (voir Clauzel, cité par Triaud, 1993) ou "naturellement indépendant" (Salifou, 1993 : 21) qui habite le nomade . Dans cette vision qui se base sur l'irréductible opposition entre sociétés lignagères et sociétés étatiques, ordre parental et ordre politique, pensée archaïque et pensée moderne, parler de "découpage" colonial au sujet des Touaregs devient hérétique, puisque ces derniers auraient depuis toujours été divisés en bandes éparses et autonomes sans aucun lien entre elles.

Est-il nécessaire d'ajouter que cette interprétation des faits est la seule admise par les gouvernements maliens et nigériens aux prises avec le problème de la rébellion touarègue ? La voix de Bourgeot se mêle, dans une parfaite osmose, aux thèses nationalistes développées aujourd'hui, de manière très offensive, dans la presse 
nigérienne, malienne et algérienne, depuis la naissance des fronts armés touaregs, et sa démarche trouve un support privilégié dans les écrits coloniaux.

Ainsi à l'anarchie touarègue est opposée "l'unité nationale (du Niger) dont la profondeur historique est plus réelle qu'on ne le dit souvent " (Bourgeot et Casajus, 1993), unité des Etats dont Bourgeot se fait l'ardent défenseur ; à propos, s'agit-il ici d'une création idéologique ou d'une réalité scientifique ?

Dans un souci semblable de légitimation de l'ordre établi, l'historien nigérien D. Hamani surenchérit en apportant à cette thèse des raffinements supplémentaires dans un article récent paru dans Niyya $\left(\mathrm{N}^{\circ} 4\right.$, avril 94), le journal de l'un des deux partis au pouvoir. Il s'applique par exemple à démontrer non seulement le bien-fondé des frontières étatiques, mais également l'opportunité de leur tracé :

“Il n’y a jamais eu, écrit-il, un monde touareg uni, ni politiquement ni économiquement ; la frontière actuelle du Niger avec l'Algérie correspond à la limite des zones d'influence du sultan d'Ayar (Aïr) et de l'Amenokal de l'Ahaggar ; la frontière entre le département de Tawa et le Mali correspond à la limite entre l'Amenokalat des Kel Nan et celui des Iwellemmeden Kel Ataram ; les Kel Gress et les Itesayan s'étaient séparés des Kel Ayar à la suite de guerres ; les Iberkorayan ont été expulsés de l'Ayar au XVIIe siècle..."

Voilà une belle démonstration de ce que cet historien pragmatique appelle luimême "reprendre le contrôle de notre histoire" (p.8). Un extrait de ce texte - écrit pour "le rétablissement de la vérité historique" contre les "inexactitudes et contre-vérités" et "la tendance pathologique à l'affabulation (qui) s'étale avec tant d'insolence" dans le Programme-Cadre transmis par la rébellion au Gouvernement - constitue le préambule du Document de base du Gouvernement du Niger pour servir aux négociations avec la rébellion, (Haut Commissariat à la restauration de la paix, Présidence de la République, avril 1994, $30 \mathrm{p}$. et annexes), confirmant la collusion entre cette thèse historique et l'action gouvernementale. Dans cette perspective, la rébellion devient "absurde" comme l'écrivent Bourgeot et Casajus (1991) et ses raisons "fallacieuses" comme le décrète, entre autres, l'historien et acteur politique nigérien A. Salifou (1993) dans son récent ouvrage sur la question touarègue au Niger.

C'est dans ce contexte où s'affrontent politiquement deux raisons historiques légitimantes, celle de l'Etat (malien ou nigérien) et celle exprimée par les fronts armés 
touaregs ${ }^{1}$ qu'est survenue - tardivement - la "mise en examen" de mes écrits qui développent pourtant depuis 1982 les analyses qui me valent aujourd'hui l'anathème.

\section{Lecture tronquée ou analphabétisme?}

Ainsi, quand j'introduis des notions que Bourgeot ne connaît pas et que le Père de Foucauld, les administrateurs coloniaux ou les linguistes n'ont pas décrites, c'est que je les ai nécessairement imaginées : par exemple tamurt, ou encore le sens politique de temust, tégézé, taghma ou tawshit. Pourtant, ces concepts apparaissent dans des ethnotextes variés dont j'ai eu l'occasion de publier de larges extraits (Claudot-Hawad 1990 ) et dont les locuteurs sont parfaitement nommés et situés. Remarquons que l'ethnologue danois Nicolaïsen (1963 : 396), bravant lui-aussi la définition du Père de Foucauld qui ne retient que le sens parental du mot tégézé, prononcé tégéhé dans l'Ahaggar, observe que "when the vassals were divided between the Kel Rela, the Taytok and the Tegehe Mellet these noble tribes became united in a sort of federation known as tegehé". Encore une invention à dénoncer.

Quand, avec beaucoup de peine, Bourgeot parvient à prendre ces notions en compte, c'est toujours d'une manière réductrice qui traduit les limites de sa propre problématique ou l'ampleur de sa malhonnêteté : par exemple, tamurt dont les contours sémantiques multiples sont décrits dans mon texte ${ }^{2}$ (p.178), devient le terme qui s'applique exclusivement à 'l'ensemble formé par les Touaregs et les autres Berbères par opposition au monde arabe ou encore occidental", choix qui permet ensuite de faire de moi l'agent d'une idéologie pan-berbère.

De même, le mot imajaghen (ou imuhagh, imushagh selon les régions) est réduit par cet auteur à l'acception particulière d'"aristocrates-guerriers" selon ses

\footnotetext{
1 ou encore par M. Dayak dans son ouvrage : Touaregs la tragédie (Lattès, 1993) vertement critiqué dans la presse par Bourgeot (Approche manichéenne d'une tragédie, Le Nouvel Afrique Asie n³7, oct. 1992, 52-53) qui lui reproche entre autre de "blanchir" la période coloniale. Malgré des conclusions divergentes, c'est pourtant suivant la même logique évolutionniste que les deux auteurs analysent le monde touareg .

2 "Le mot tamurt désigne un ensemble de personnes possédant la même culture, la même langue ou la même mythologie fondatrice. L'accent est mis dans cette notion sur l'affinité culturelle. Les liens définissant la collectivité ainsi nommée peuvent exister actuellement ou appartenir au passé. Par exemple, les Touaregs considèrent qu'autrefois les descendants de Noé, devenus plus tard les Berbères, Arabes, Ethiopiens, Peuls, Egyptiens et Juifs constituaient la même tamurt... A une échelle plus réduite, les Touaregs et les Peuls ou encore les Touaregs et les Maures peuvent être présentés comme membres d'une ancienne tamurt..."
} 
termes, alors qu'il désigne également - c'est explicitement dit dans mon texte, voir p. 99 - toutes les personnes de culture touarègue sans considération de catégorie sociale : cette fois c'est de nostalgie féodale, de création d'un "homo targuicus, être supérieur", dont je me rends coupable. Pourtant, le sens large de ce terme a été relevé par de nombreux observateurs. S'agirait-il d'une hallucination collective ? Je renvoie Bourgeot par exemple à Duveyrier (1864 : 317), à Benhazera (1908: 84), “Ceux que nous avons coutume d'appeler les Touaregs, d'après le nom que leur a donné les Arabes, s'appellent en réalité Imouhar', Imochar' ou Imohar' selon les dialectes", ou à Foucauld qu'il affectionne (Dict.II : 534) parlant de "ceux que les Arabes appellent 'Touareg' et que les Kel -Ahaggar appellent Imouhâr"”, ou à Richer (1924 : 18), ou encore à Ghubayd ag Alawjeli (1980 : 126) qui pour le terme amajagh/imajaghen donne dans son lexique la définition suivante : "Touareg // Touareg noble (membre de la classe des nobles // h./an. brave, courageux // f. sg.\& langue touarègue // langue berbère".

Comme désignation identitaire des Touaregs, Bourgeot retient exclusivement l'appellation Kel tamashaq (ou Kel tamahaq, Kel tamajaq selon les parlers), qui signifie "les touarégophones, ceux de la langue touarègue". Or tamashaq ou tamajaq est le féminin singulier du terme imajaghen incriminé plus haut. Dans la définition restrictive qu'en donne Bourgeot, il signifierait donc à la fois "femme aristocrate-guerrière" et "langue que parlent l'ensemble des Touaregs". On se serait attendu à ce que tamajaq désigne la langue des seuls aristocrates-guerriers. Malgré lui, Bourgeot restitue à ce terme le signifié large qu'il cherchait à oblitérer. Le choix de Kel tamashaq comme unique appellation identitaire de l'ensemble touareg n'est certes pas innocent : elle dénie aux Touaregs la conscience d'appartenir à un ensemble autre que linguistique. L'instrumentalisation politique de Kel tamashaq par Bourgeot me paraît d'autant plus évidente que cette appellation n'est utilisée, de préférence à imajaghen ou à fréquence égale, que très localement, uniquement par trois groupes touaregs qui se trouvaient sous la dépendance des Iwellemmeden de l'ouest, c'est-à-dire les Kel Adagh, les Kel Intessar et les Imedédaghen. Par contre, dans le reste de la Tademekkat ainsi que dans l'Ajjer, l'Ahaggar, l'Aïr ou la Tagaraygarayt, c'est-à-dire dans la grande majorité du monde touareg, c'est bien le terme imajaghen (imuhagh, imushagh) qui est utilisé avant tout autre.

Pour prouver que les tributaires (imghad) n'ont pas les mêmes référents identitaires que les nobles et ne se sentent pas concernés par la temujagha ("targuité, 
humanité") $)^{3}$, Bourgeot a de nouveau recours au Dictionnaire du Père de Foucauld - que ne fait-il ses enquêtes lui-même - et en extrait tamegheda. Cette notion, loin de constituer un concept identitaire revendiqué par les dépendants, a une connotation péjorative et s'applique à toute personne, quelle que soit sa catégorie sociale, dont la conduite apparaît contraire à l'idéal prôné par la société. Cet idéal est bien sûr — et très banalement - la noblesse du comportement et de l'attitude que cherche à atteindre tout individu quel qu'il soit, considéré en marche vers l'accomplissement de soi, aucune position sociale ni individuelle n'apparaîssant immuable ou acquise une fois pour toutes. Dire à un amghid qu'il a de la tamegheda est une véritable provocation : Bourgeot devrait mettre ce concept en application pour en mesurer l'effet. Cela reviendrait par exemple à traiter un nigérien de $b u z u$, terme qui en haoussa signifie “esclave, carpette" et sert au Niger depuis l'indépendance à désigner les Touaregs, bien qu'ils soient estampillés "nigériens" sur leur carte d'identité.

Autre exemple, la polysémie de temust - qui désigne à la fois le corps social et l'identité individuelle (que les locuteurs cultivés appellent aussi temusa, terme de la même racine) - désempare totalement ce chroniqueur qui pense qu'à un seul terme doit nécessairement correspondre une seule signification. Sens figuré, sens propre, dénotation, connotation, tout cela est de l'idéologie pour Bourgeot qui s'emploie à trier exclusivement ce qui peut alimenter ses thèses. Ainsi, lorsque - à partir, je le souligne, des récits historiques recueillis dans l'Aïr sur la guerre de Kaosen contre l'armée coloniale française - je décris la notion de temust comme un ensemble (dont je m'efforce de montrer les formes et les transformations) où s'articulaient les cinq grandes confédérations touarègues, je crée de l'ethnicité, j'invente un peuple et une société qui n'existent pas, bref je fabrique de l'identité discriminatoire. De toute évidence, là encore, Bourgeot n'a pas même lu l'introduction et encore moins la fin de l'ouvrage dont il était censé rendre compte. Les interrelations et le brassage multiples d'unités sociales hétérogènes au sein de formations politiques, sociales et culturelles, beaucoup plus vastes que l'espace tribal ou ethnique où le regard colonial les a généralement confinées, est au centre de ma problématique (Claudot-Hawad 1993b, 1993c, 1993d). Je me sens d'ailleurs à ce sujet beaucoup plus en affinité avec l'approche

\footnotetext{
3 On se demande ce que deviennent dans ce schéma les forgerons, les religieux, les affranchis et les esclaves.
} 
théorique récente des anthropologues de l'Amazonie (voir L’Homme, 1993, n¹26-128) qu'avec celles des spécialistes du Sahara et du Sahel.

Enfin, autre création ex-nihilo dont je me rendrais coupable : la notion de temust $n$ imajaghen, "peuple ou nation des Touaregs", concept des plus banals dont l'occurence est courante dans les multiples enquêtes que j'ai réalisées sur l'organisation sociale et politique touarègues et notamment dans les récits variés sur la guerre de Kaosen, largement cités dans mes écrits et qui ont été enregistrés en 1989 et 1990 dans l'Aïr, auprès de témoins ou acteurs directs de cette période, tels que Baba ag Moussa, Fakando ag Cheikho, Ghaïshena welet Akédima des Ikazkazen. J'ajoute qu'aucun de ces nonagénaires ne fait partie des ishumar où se recrutent les membres des fronts armés actuels et auxquels Bourgeot attribue la création de cette notion, tout récemment décalquée d'après lui sur les concepts politiques "modernistes".

A cette notion de temust, à laquelle Bourgeot dénie le sens d'identité collective, j'aurais donc adjoint $n$ imajaghen, qui pour lui signifie "celle des aristocrates-guerriers". Voilà le signifié des termes à nouveau parqué dans l'individuel et l'aristocratique. Quant au signifiant, la preuve de son caractère factice est fournie par Casajus qui invoque les règles grammaticales touarègues et l'état d'annexion qu'elles imposent au complément de nom masculin pluriel, privé alors du $i$ initial, ce qui donne par assimilation consonantique : temust emmajaghen. Ainsi, je n'ai pu "entendre" cette expression et la conclusion est à nouveau que je l'ai inventée.

Cette objection appelle une double réponse. La première est que si l'amalgame phonétique cité est couramment pratiqué dans certains milieux, il est jugé dans d'autres comme un usage négligé, vulgaire et inadmissible de la langue. Mes interlocuteurs articulaient bien temust $n$ imajaghen. La deuxième est que si je m'étais effectivement trouvée face à la réalisation sonore décrite par Casajus et formalisée notamment par les Petites Soeurs de Foucauld dans leur ouvrage intitulé Initiation à la langue des Touaregs de l'Ä̈r (Agadez, 1968), je l'aurais traitée en bonne saussurienne comme une "variante contextuelle", c'est-à-dire comme l'une des réalisations sonores possibles d'un phonème, d'une part, en fonction de son entourage consonantique et vocalique ( $n$ devient em) et d'un morphème, d'autre part, marquant le pluriel du nom masculin (composé d'un préfixe et d'un suffixei-en qui se mue en suffixe-en) en fonction du contexte morphologique et phonétique. A une transcription phonétique qui note toutes les différences que perçoit le transcripteur, je préfère l'option phonologique et 
morphologique, qui retient les traits qu'une analyse de la langue a révélé distinctifs ou dotés d'une fonction linguistique.

A ce propos, pourquoi Bourgeot n'adresse-t-il pas la même critique à Klute dont le texte cité par lui produit une transcription identique à la mienne pour temust $n$ imajaghen? A transcription similaire correspondraient donc un bon et un mauvais filtrage phonétique, une élocution authentique et une élocution fictive ?

Que dire enfin du désarroi psychologique de cet auteur lorsqu'il découvre la métaphore du corps politique touareg non seulement affublé de multiples cuisses, bassins et poignets mais en plus asexué et acéphale ? Voilà qui ne correspond ni au seul modèle bien concret du "corps" que parvient à concevoir Bourgeot, ni à sa représentation d'une formation politique fédérale qui dans son esprit doit nécessairement être chapeautée par un "chef suprême". Pour la critique de cette conception étroitement ethnocentrique, je renvoie à la réponse que j'ai déjà longuement formulée sur le même sujet dans la revue Politique Africaine $\mathrm{n}^{\circ} 50$, ainsi qu'à l'introduction de l'ouvrage Le politique dans l'histoire touarègue (1993b) pour l'exposé de ma problématique.

Je rappelle qu'en dépit des assertions gratuites de Bourgeot, j'ai montré concrètement (Claudot-Hawad 1990, 1993b) comment ce concept du "corps de la société" est mis en action à travers l'assemblée suscitée par Kaosen à Agadez au début du siècle et comment la fonction du chef-arbitre de l'Aïr (amenukal) institutionnalise la gestion des rapports interconfédéraux. Mon enquête sur le déroulement de la fête de Gani illustre à son tour la mise en scène symbolique de ce corps politique. Enfin, il existe de multiples versions de l'histoire des origines qui relient au sein d'un réseau généalogique unifiant — remodelé au grè des recompositions politiques — les différentes composantes de la société touarègue, articulant sur le mode parental non seulement les confédérations (ettebel) que Bourgeot considère comme des unités politiques autonomes sans aucun lien entre elles, mais aussi les fédérations de confédérations (tégézé) — voir par exemple Benhazera 1908 : 92, 173 au sujet des trois ettebel (pl. ettebelen) Kel Ghela, Taytoq et Tégéhé Mellet ; Nicolaisen 1963 : 405-406 au sujet des Kel Ahaggar et Iwellemmeden, Duveyrier 1864 : 322 au sujet des Kel Ahaggar et Kel Ajjer...

Selon la même interprétation aliénée, Bourgeot m'accuse de confondre légende et histoire au Mali, alors que je parle clairement de la "conscience historique" de mes interlocuteurs — c'est-à-dire de la conscience qu'ils ont d'avoir une histoire — sans 
préjuger bien entendu de la "vérité" de cette histoire pas plus d'ailleurs que de celle de l'histoire "nationale" construite du point de vue de l'Etat malien.

Toujours disqualifiant, un nouveau jugement de valeur s'épanche à propos du récit de Nukh décrivant Koseilata comme l'ancêtre des Imedédaghen, m'accusant de confondre, avec la malhonnêteté qui me caractériserait, une légende individuelle (historiette) et une épopée historique. Je me trouve patiente de répondre à tant d'ignorance arrogante. Le thème de Koseilata comme ancêtre des Imedédaghen est le plus riche, le plus connu, le plus incontournable de l'histoire orale de l'Adagh, de l'Awza et du Gourma (mais seul des auteurs "lucides" comme les aime Bourgeot sauraient être crédités de véracité : renvoyons le donc par exemple aux défunts Barth, 1858, t.V : 556, Benhazera, 1908 : 222 ou Richer, $1924: 39$ ).

Enfin, pour prouver que la mémoire historique touarègue est des plus "confuses", amalgamant espace et temps, n'égrenant que d'invraisemblables sornettes, cet auteur se réfère doctement à Ibn Khaldoun, toujours à propos du personnage de Koseilata. Est-il possible que Bourgeot ne se soit jamais aperçu que dans l'ouvrage d'Ibn Khaldoun auquel il accorde le statut "historique", tout comme dans celui d'El-Bekri qu'il me reproche plus loin d'avoir omis de citer, les personnages sont doués d'ubiquité autant que dans 1'histoire orale qui d'ailleurs leur a servi de support ? Prenons l'exemple d'ElBekri qui fait de l'Aurès la demeure de la Kahéna et d'une manière plus générale la place forte de l'opposition berbère - puisque Abu Yezid (né à Essuk dans l'Adagh en 885, de mère touarègue d'extraction servile et de père originaire du Nefzawa, d'après Mauny, 1961 : 117) ou Koseila (installé dans le Sous avec les Awreba selon Ibn Khaldoun) y conduiront leur révolte. Quelque paragraphes plus loin, cependant, El-Bekri précise que c'est à Ghadamès dans l'Ajjer que l'on voit les souterrains que la Kahéna aurait employés comme prisons (340), ou encore que Koseila était roi de Tobna, c'est-à-dire Batna, capitale des Aurès (108). Entre l'Adagh et l'Ajjer (pays touareg), le Sous et les Aurès, l'espace et le temps, loin d'être cloisonnés, apparaîssent dans ces récits comme un continuum. La forme du rapport espace/temps ainsi que l'organisation du discours, ses pôles signifiants, ses thèmes récurrents, ses critères de véracité se révèlent pertinents, au même titre que le sont ceux du discours historique moderne comme l'a montré Lina Brock à propos des récits et écrits sur la révolte de 1917 chez Touaregs Kel Denneg (1990).

Je suis surprise enfin que Bourgeot, qui affectionne si vigoureusement les travaux de terrain "sous l'accacia" (Bourgeot, 1993), accorde tant d'importance à El 
Bekri qui a rédigé ses manuscrits sur l'Afrique sans quitter les palais andalous, ou encore à Ibn Khaldoun qui sur la route du sud n'a jamais dépassé la palmeraie de Biskra.

Avec la monotonie d'un leit-motiv, la vision réductrice de Bourgeot poursuit son opération mutilante : lorsque je dis que la référence à l'islam ne permet pas d'expliquer de manière satisfaisante la structure de certains rituels et la représentation du monde qu'ils illustrent, je ne nie évidemment pas l'islamisation des Touaregs, mais je situe l'espace d'efficience de l'islam ailleurs.

Faux procès toujours au sujet de la définition unilinéaire de la tawshit, que je qualifie d"officielle" dans le sens de représentation normative par opposition à la composition effective de ce groupe qui s'avère bilatérale. Rassurons Bourgeot, il n'existe aucune anthropologie touarègue officielle, à part la sienne.

Finalement, après ces permanentes attaques contre les thèmes et les problématiques de recherche lancés par notre équipe (par exemple sur les biens indivis, l'identité, les ishumar, l'école coloniale...), on se demande pourquoi Bourgeot continue avec tant de constance à les récupérer et à les recycler pour son propre compte. On s'attendrait à plus d'autonomie et d'originalité de sa part.

\section{« To be or not to be, there is the rub » (Shakespeare)}

Etre ou ne pas être, voilà une question que ne se pose pas Bourgeot au sujet des matériaux sur lesquels je fonde mes analyses. Son discours inquisiteur qui se satisfait d'affirmer sans jamais démontrer s'appuie en effet sur le postulat de la non-existence de mes enquêtes de terrain. Quid de mes données réunies dans l'Ahaggar, l'Adagh, le Gourma, l'Awza, l'Aïr, etc, qui ont fait l'objet de tant de publications, quid des interlocuteurs ou auteurs cités nommément dans l'ouvrage incriminé tels que le vieux Bey des Kel Essuk torturé à mort en 1990 par l'armée malienne, Nukh et Ekhya des Imedédaghen Kel Elakad, Khumaydata des Imedédaghen de Gossi, Khamada des Kel Ehan Ishagaghen, Ikhna des Imedédaghen de Tésit, Mohamed ag Intenekad des Idnan, Mohamed des Imédedaghen de Tombouctou, Ghaïchena des Ikazkazen, Mohamed Ali ag Ataher des Kel Intessar, etc ? Mais à quoi bon puisque les voix "endogènes" ne sont que faux témoignages et fables personnelles dans le procès que mène Bourgeot. 
Ainsi, prétend-il que ma démarche repose exclusivement sur l'"egovision" d'un informateur unique, allusion à mon époux Hawad auquel je rendrais un "hommage appuyé". L'affaire de l'"historiette" individuelle rebondit et le schéma évolutionniste structure si bien la pensée de Bourgeot qu'il lui sert à nouveau d'attelle dans le registre diffamatoire. En effet, quel autre rapport intellectuel pourrait avoir une ethnologue avec un indigène si ce n'est celui qui lie le penseur à l'informateur ? Je précise que sur les douze articles réunis dans l'ouvrage qui nous doit l'échauffement de Bourgeot, seuls trois d'entre eux font référence à Hawad en sa qualité d'auteur au sujet des tifinagh cursifs et de la calligraphie qu'il pratique, au sujet de la littérature touarègue contemporaine (Hawad est avec Alkuni le seul auteur littéraire touareg publié à ma connaissance) et au sujet de la fête de Gani sur laquelle il apporte un témoignage d'une grande richesse qui m'a incitée à mener une enquête sur la question en 1990 dans l'Aïr.

Le reproche corollaire que Bourgeot m'adresse de ne pas citer les auteurs “dont la compétence est reconnu par la communauté scientifique" - s'agit-il d'une marque déposée? - laisse apparaître du dépit de la part de cet auteur de compte-rendu ; le dépit de ne pas être suffisamment nommé peut-être ? En effet, ma bibliographie n'a aucunement la prétention de livrer une documentation exhaustive sur les Touaregs - il existe pour cela d'excellents ouvrages bibliographiques, voir Brenier-Estrine, Chaker, Leupen - mais elle a pour fonction de donner les références des titres cités in-texto.

Un autre procédé dévaluant consiste à affirmer que ma démarche ainsi que celle de Hawad, tant qu'à faire, ne sont ni l'une ni l'autre originales : nous serions des pirates. Ainsi, je m'inspirerais, sans jamais citer mes sources évidemment, de Duveyrier au sujet du concept de "nation" appliqué aux Touaregs, de Casajus pour l'interprétation symbolique - je me défends d'avoir quoi que ce soit de commun avec la vision sans rigueur de Casajus (voir à ce sujet le compte-rendu critique de son ouvrage dans l'Annuaire de l'Afrique du Nord, XXIX, 1986, 867-875) — et enfin de la "vision personnelle d'un Touareg, auteur de poésies"(sic).

"Si ce n'est toi, c'est donc ton frère" ou ton époux... La raffle disqualifiante concerne également Hawad, créateur de tifinagh cursifs ainsi que d'une calligraphie tifinagh : il ne serait d'après Bourgeot que "l'adepte" de Ghubeyd ag Alawjaly pour l'écriture. Pourquoi Bourgeot qui a une appréhension si concrète de la réalité ne compare-t-il pas les signes de Hawad et ceux produits plus tard par Ghubeyd (et publiés in Claudot-Hawad 1988) dans des circonstances dont ce dernier ne faisait d'ailleurs aucun mystère lors de notre entrevue en janvier 1988 ? Pourquoi n'interroge-t-il pas 
Ghubeyd lui-même qui d'ailleurs n'a jamais rédigé ses ouvrages autrement qu'en caractères latins ? Comment une écriture qui n'est pas rodée par l'usage peut-elle soudain devenir cursive ? Voilà un miracle scientifique.

Hawad copierait enfin la calligraphie arabe ésotérique que d'ailleurs "les Kel Tamashäq ne peuvent pas lire" - belle preuve d'ignorance quant à l'oeuvre de Hawad et à sa calligraphie qui peut seulement être ésotérique du moment que Bourgeot est incapable de l'interpréter ; renvoyons-le à l'abondante bibliographie sur la question (notamment Hawad 1987, 1992, introduction de 1993c, Penel 1990), encore que la pensée philosophique d'un indigène "auteur de poésies" n'ait aucun poids face à celle de l'administrateur colonial, du missionnaire, ou de l'universitaire patenté qui seuls, selon cet auteur, savent évaluer le "bon" sens des choses.

Finalement, pour rendre compte de la "dérive inquiétante" de mes travaux emplis de "contre-vérités" (Casajus, Rapport de la session d'automne 1993 sur mes activités de recherche, section $38 \mathrm{du}$ CNRS), Bourgeot et Casajus ont une explication qui relève de "faits privés" : en effet, je suis mariée à un Touareg, donc obligatoirement partiale. C'est le délit d'indigénisation.

Aveuglement ? Soif de pouvoir ? Transition doctrinale douloureuse ? Suppression de la manne des sauvages à expertiser et à développer ? Bourgeot est en crise. Son école de pensée évolutionniste, cautionnée par les gouvernements saharosahéliens qui veulent en finir avec le problème touareg, est contestée par des brebis galeuses, certaines, indigènes de surcroît (voir par exemple les articles du $n^{\circ} 51$ de Politique Africaine sur les intellectuels africains).

La position caricaturale de Bourgeot démontre les difficultés qu'éprouve la science à s'écarter du paradigme du pouvoir politique. Que l'histoire soit un enjeu politique est un truisme. Mais lorsque cette considération, par ailleurs intéressante car elle devrait engager à la vigilance, n'est appliquée qu'aux versions qui contredisent l'histoire officielle, elle devient l'instrument même du dogme et de la censure. Est-ce que la censure a un sens dans nos disciplines ? Notre rôle n'est-il pas de mettre à jour d'autres intelligibilités historiques que celles que légitiment les pouvoirs en place, afin, peut-être, d'être moins surpris par les événements qui feront l'histoire de demain ?

Posant le problème de la vérité en histoire dans une remarquable étude sur la révolte de 1917 chez les Touaregs Kel Denneg, Lina Brock (1990) disait : "Finalement, c'est en assemblant les récits qui représentent des prises de position importantes dans 
une société donnée - (ou dans les) différentes sociétés en présence - que nous pouvons entendre non pas une seule voix mais la conversation à laquelle ces voix prennent part".

Jugeons-en. Beaucoup de chemin reste à parcourir pour restituer cette discussion et faire admettre la pluralité des voix contradictoires et complémentaires qui seules font l'histoire.

\section{Bibliographie}

\section{El-Bekri, Abou-Obeïd}

1965, Description de l'Afrique septentrionale, Trad. M. de Slane, Paris, Maisonneuve, 1965.

Bourgeot, A

1990, Identité touarègue : de l'aristocratie à la révolution, Etudes rurales, nº $120,129-162$.

1992, L'enjeu politique de l'histoire : vision idéologique des événements touaregs (1990-1992), Politique Africaine $\mathrm{n}^{\circ} 48$.

Bourgeot, A. et Casajus, D

1992, Les Touaregs piégés par la guerre, Libération 27 juillet, p.2.

1993, Les vertus de la démocratie, Le Monde des débats, juillet-août.

Brenier-Estrine, $\mathrm{C}$.

1994, Bibliographie berbère annotée 1992-1993, Travaux et documents de l'IREMAM nº16, Aix-enProvence, $152 \mathrm{p}$.

Brock, L.

1990, Histoire, tradition orale et résistance : la révolte de 1917 chez les Kel Denneg, REMMM $\mathrm{n}^{\circ}$ 57, Edisud, Aix-en-Provence, 49-76.

Chaker, S.(ed.)

1988, Etudes touarègues, Bilan des recherches en sciences sociales, Edisud, Aix-en-Provence, $192 \mathrm{p}$.

Claudot-Hawad, H.

1988, Tifinagh, de la plume à l'imprimante, Travaux du LAPMO, Aix-en-Pce, 225-227.

1990, Honneur et politique : les choix stratégiques des Touaregs pendant la colonisation, in Touaregs, exil et résistance, REMMM n 57, Edisud, Aix-en-Provence, 11-47.

1992, Bandits, rebelles et partisans : vision plurielle des événements touaregs de 1990-1992, Politique Africaine $\mathrm{n}^{\circ} 46$.

1993a, Histoire d'un enjeu politique : La vision évolutionniste des événements touaregs 1990-1992, Politique Africaine $\mathrm{n}^{\circ} 50,132-140$.

1993b, Le politique chez les Touaregs: un ordre absent, insoupçonné ou occulté, in H. Claudot-Hawad (ed.), Le politique dans l'histoire touarègue, Cahiers de l'IREMAM, n4, 198 p. 5-11

1993c, La coutume absente ou les métamorphoses contemporaines du politique chez les Touaregs, in op. cité, 67-85

1993d, Les fibres synthétiques de la culture, Autrement, sous presse.

Claudot-Hawad H. et Hawad, M.

1982, Coups et contre-coups : l'honneur en jeu chez les Touaregs, Annuaire d'Afrique du Nord XXI, 793-808.

Clauzel, J.

1992, L'administration coloniale française et les sociétés nomades dans l'ancienne Afrique occidentale française, Politique africaine, $\mathrm{n}^{\circ} 46,99-116$.

Foucauld, Ch. de

1951-52, Dictionnaire touareg-français, Dialecte de l'Ahaggar, Paris, Imprimerie Nationale, 4 t. 
Galle, E.

1987, L'homme vivant de Wole Soyinka, Silex, Paris, 271 p.

Hamani, D.

1994, Une gigantesque falsification de l'histoire, Niyya $\mathrm{n}^{\circ} 4$, avril, Niamey, 5-8.

Hawad

1987, Mots, traits, signes, Podio n ${ }^{\circ}$, Grasse.

1990, La teshumara, antidote de l'Etat, REMMM n ${ }^{\circ} 57,123-140$.

1992a, Entretien réalisé par G. Dessons et X. Person, in coll., Hawad, Office du Livre en PoitouCharentes, Poitiers, 17-28.

1992b, Lettre d'un homme touareg à une femme haoussa, Le Républicain Niamey, 20 février.

1993a, Une identité dans le sillage de l'infini, Les Lettres françaises n³0, mars, 14-15.

1993b, Hachis touareg pour diners officiels, Libération, 12 janvier

1993c, Horizon van een nomade (Calligraphies), Ed. Nominoë, Rotterdam.

Klute, G.

A paraître, Hostilités et alliances. L'aspect historique de la dissidence dans le mouvement rebelle des Touaregs du Mali, Cahiers d'Etudes Africaine.

Leupen, A.H.A.

1978, Bibliographie des populations touarègues, Afrika-Studiecentrum, Leyde, $240 \mathrm{p}$.

Nicolaisen, J.

1963 Ecology and culture of the pastoral Tuareg - With particuler reference to the Tuareg of Penel, J.D Ahaggar and Ayr, National Museum, Copenhagen, 548 p.

1990, Entretien du 4/2/1990 avec Hawad à Niamey, in Rencontre, J.D. Penel et A. Maïlele, Editions du Ténéré, Niamey, 201-234.

Présidence de la République du Niger, Haut Commissariat à la restauration de la paix

avril 1994, Document de base du Gouvernement du Niger pour servir aux négociations avec la rébellion, $30 \mathrm{p}$ et annexes.

Richer, A.

1924, Les Oulliminden, Paris, Larose, 359 p.

Salifou, A.

1993, La question touarègue au Niger, Karthala, 203 p.

Triaud, J.L.

1993, Le temps des "bandits", in Bernus, Boilley, Clauzel, Triaud (ed.), Nomades et commandants, Karthala, 15-19. 